\title{
Effect of Vibration on the Melting of Phase Change Material inside a Cylindrical Enclosure
}

\author{
Mohammadhossein Hajiyan, Manar Al-Jethelah, Yazeed Alomair, Muath Alomair, Syeda Tasnim, Shohel \\ Mahmud \\ School of Engineering, University of Guelph \\ 50 Stone Rd E, Guelph Ontario Canada, N1G 2W1 \\ mhajiyan@uoguelph.ca; maljethe@uoguelph.ca; yazeed@uoguelph.ca; alomairm@uoguelph.ca; stasnim@uoguelph.ca; \\ smahmud@uoguelph.ca
}

\begin{abstract}
The present study reports a novel numerical work on the melting of phase change material (PCM) inside a cylindrical enclosure under the application of vibration. No such work is available in the existing literature. To simulate the melting of PCM inside a vertical cylindrical enclosure, the governing equations, namely, the conservation of mass, momentum, and energy equations are solved. In addition to that enthalpy-porosity method is used to model phase change of PCM. COMSOL Multiphysics software is used to simulate the melting behaviour of a PCM during vibration conditions. The objective of the present work is to observe the melting behaviour under the application of vibration. Melting behaviour of PCM inside a vertical cylindrical enclosure has been simulated first without vibration for comparison purpose. It has been observed that vibration affects the melting behaviour significantly. This study is applicable to battery thermal management for the hybrid vehicles where PCM can be used for controlling the temperature of the battery.
\end{abstract}

Keywords: Phase Change Material, Cylindrical Enclosure, Numerical Simulation, Vibration, Melting.

\section{Introduction}

Thermal energy storage (TES) has been the main goal for many engineering applications globally over the last 30 years due to environmental pollutions and energy crisis. Numerous experimental, numerical and theoretical studies have been conducted to enhance the efficiency of energy storage systems, by increasing the thermal conductivity, heat capacitance or optimizing the geometry of the systems. PCMs are used for storing thermal energy in terms of sensible and latent heat. PCM based latent heat thermal energy storage (LHTES) system contributes significantly to the efficient use and storing of waste heat and renewable energy and the stored energy is can be used for many applications, such as, domestic hot water, air conditioning, storing foods and medications, refrigeration and electronic devices cooling [1]. Lorente et al. [2] numerically and analytically investigated melting performance of paraffin wax in a vertical cylinder enclosure. The melting point temperature of the PCM is $29.85^{\circ} \mathrm{C}$. The analytical results showed good agreement with the simulation results. Esen et al. [3] numerically and analytically investigated melting of different PCMs in a cylinder enclosure for domestic heating. The results showed that the melting time of a PCM depends on geometric parameters, thermal parameters and thermo-physical properties of the PCM. Kashani et al. [4] numerically studied the performance of heat transfer of PCM with different concentrations of copper nanoparticles in rectangular and cylindrical enclosures. The results showed that the cylindrical container of the same nano-PCM takes two times longer period than rectangular container. Sciacovelli et al. [5] numerically investigated the performance of PCM melting in a vertical cylinder. The authors studied the heat transfer enhancement by adding different volume fractions of copper nanoparticles from $0 \%$ to $4 \%$. The authors found that $4 \%$ copper nanoparticles reduced the melting period compared to other volume fractions studied. Singh et al. [6] numerically studied the performance of enhancing heat transfer by adding metal foam in a cylinder for concentrated solar power systems. Melting and solidification analyses were performed on Sodium Chloride PCM that has a melting point of $800^{\circ} \mathrm{C}$. The numerical results showed an enhancement of heat transfer and reduced heat transfer fluid (HTF) pipes bypasses by a factor of 12 when metal foam is added. Liu et al. [7] performed numerical analysis on melting of heat transfer with adding heat sink inside a cylindrical container. They found that the melting time of the PCM was reduced with smaller outer layer heat sink diameter. Chiu and Martin [8] investigated melting and solidification of heat transfer 
performance of PCM in one and multi-stage in a cylindrical shell. In the numerical results, multi-stage system showed significant improvement during melting process. Bilir and Ilken [9] numerically studied the solidification time of a liquid PCM in cylindrical and spherical containers. In the mathematical formulation, the enthalpy method was used. The governing dimensionless equations were solved to obtain the total solidification time of the PCM. Archibold et al. [11] performed a numerical investigation of the melting process of a relatively high temperature PCM in partially filled spherical containers. Results showed heat transfer enhancement with increasing the Grashof number. For a fixed Grashof number, the melting rate of the PCM was faster with increasing the Stefan number. Karthikeyan et al. [12] performed an analytical study on packed bed storage unit filled with PCM in a spherical capsule for low temperature solar air heating applications. The results showed that increasing the heat transfer fluid (HTF) flow rate and higher temperature difference between the HTF and the PCM temperature are crucial factors to increase the rate of heat transfer with small sized capsules during the charging process. Sattari et al. [13] numerically analysed the melting process of PCMs in an encapsulated spherical container. The results showed a thermally stable layer on the top of the sphere whereas, disturbances exist at the bottom of the sphere because of the natural convection. Decreasing the diameter of the sphere and/or increasing the surface temperature of the encapsulated sphere increased the melting rate of the PCM. Karthikeyan and Velraj [14] compared and analysed several mathematical models on packed bed storage unit filled with PCM in a spherical capsule numerically. In the mathematical formulation, the enthalpy method is used to study the phase change behaviour of the PCM within a range of temperatures. The effects of using water and air as HTF are investigated as well. Two mathematical models were recommended for different HTFs. Archibold et al. [15] studied the effect of radiative heat transfer during melting process of a high temperature PCM in a spherical container. The results showed that the melting process of the PCM is enhanced by the presence of thermal radiation. Yang et al. [16] performed a thermal analysis of a solar storage packed bed using encapsulated spherical container filled with PCM having different melting points. The HTF inlet temperature was fixed for all three different melting points of the PCMs. Results showed a good agreement with previous studies. Ismail and Henriquez [17] studied the solidification of PCM in an encapsulated spherical container. The mathematical model is based on pure conduction. The results of the model were validated with previous and similar models. Based on the above literature review, none of the studies has investigated the melting of PCM melting under vibration conditions. The aim of this novel study is to investigate the melting behaviour of PCM under vibration conditions numerically.

\section{Mathematical Modelling}

In this study, it is assumed that the liquid form of the PCM is a Newtonian incompressible fluid and laminar flow is considered for liquid phase PCM. Figure 1 shows the schematic view of cylindrical enclosure with specified dimensions and mesh distribution. Note that all the surfaces are exposed to the hot temperature where the melting occurs and there is no insulation. To simulate the system, the governing equations, namely, the conservation of mass, momentum, and energy are solved numerically as follows: 

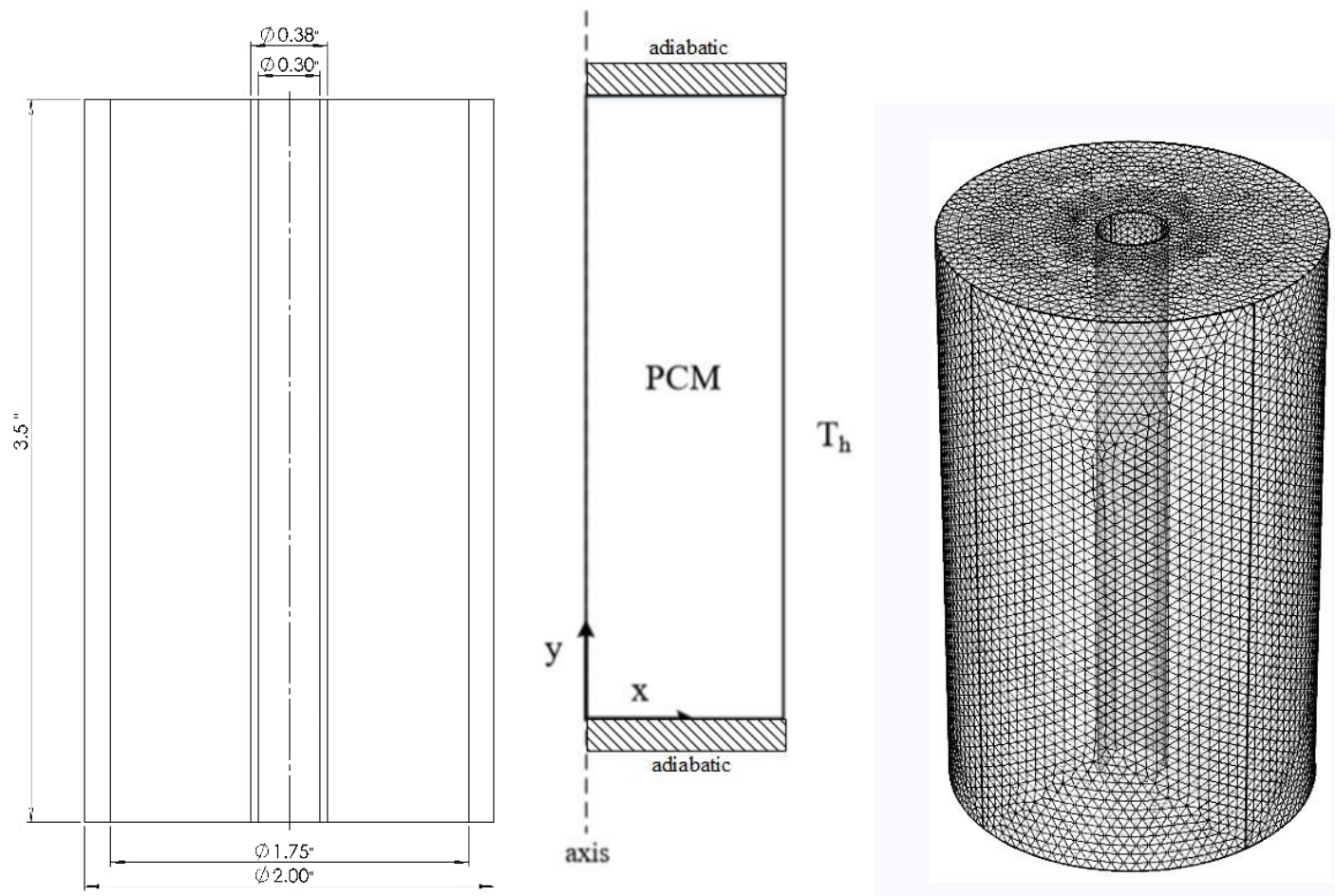

Fig. 1: Cross section, axisymmetric and 3D view of mesh distribution for cylindrical enclosure filled with PCM.

Mass Conservation (liquid):

Momentum Conservation (liquid):

$$
\frac{\partial \rho_{n f}}{\partial t}+\frac{\partial\left(\rho_{n f} u\right)}{\partial x}+\frac{\partial\left(\rho_{n f} \nu\right)}{\partial y}=0
$$

$$
\begin{gathered}
\frac{\partial u}{\partial t}+u \frac{\partial u}{\partial x}+v \frac{\partial u}{\partial y}=\frac{1}{\rho_{n f}}\left[-\frac{\partial p}{\partial x}+\mu_{n f}\left(\frac{\partial^{2} u}{\partial x^{2}}+\frac{\partial^{2} u}{\partial y^{2}}\right)\right] \\
\frac{\partial v}{\partial t}+u \frac{\partial v}{\partial x}+v \frac{\partial v}{\partial y}=\frac{1}{\rho_{n f}}\left[-\frac{\partial p}{\partial y}+\mu_{n f}\left(\frac{\partial^{2} v}{\partial x^{2}}+\frac{\partial^{2} v}{\partial y^{2}}\right)+g^{*}(t)(\rho \beta)_{n f}\left(T-T_{m}\right)\right]
\end{gathered}
$$

Energy Conservation (liquid)

$$
\frac{\partial T}{\partial t}+u \frac{\partial T}{\partial x}+v \frac{\partial T}{\partial y}=\frac{k_{n f}}{\left(\rho c_{p}\right)_{n f}}\left[\frac{\partial^{2} T}{\partial x^{2}}+\frac{\partial^{2} T}{\partial y^{2}}\right]
$$

Energy Conservation (solid):

$$
\frac{\partial T}{\partial t}=\frac{k_{n f}}{\left(\rho c_{p}\right)_{n f}}\left[\frac{\partial^{2} T}{\partial x^{2}}+\frac{\partial^{2} T}{\partial y^{2}}\right],
$$

where $\rho_{n f}$ is the density of the nano-PCM, $t$ is time, $u$ is velocity component in the $x$-direction, $v$ is velocity component in the $y$-direction, $p$ is pressure, $\mu_{n f}$ is dynamic viscosity of nano-PCM, $g$ is gravitational acceleration, $\beta_{n f}$ is the coefficient of thermal expansion of the liquid nano-PCM, $T$ is temperature, $T_{m}$ is melting temperature of the nano-PCM, $k_{n f}$ is thermal conductivity of the nano-PCM, and $c_{p_{n f}}$ is specific heat at constant pressure of the nano-PCM. 
Note that in Eq. (3) the gravity term $\mathbf{g}^{*}(\mathbf{t})$ has been modified and considered as a cos function of time as follow:

$$
\mathbf{g}^{*}(\mathbf{t})=\mathbf{g}_{0}(\mathbf{1}+\mathbf{A} \cos (\boldsymbol{\omega} \pi \mathbf{t})+\varphi)
$$

where $g_{0}$ is constant gravity, $A$ is the scaling parameter, $\omega$ is the frequency of oscillation, and $\varphi$ is the phase of the cos function which changes the direction over time. In this study, the magnitude of amplitude $(A)$ is considered to be 2.5 and phase is $1(\varphi=\mathbf{1})$. Thus, $\omega$ is changing in this study to generate oscillation in gravity term which directly affects the buoyancy force applied to the PCM. Figure 2 shows the oscillation used in this study as a function of time for 100 seconds. Note that this pattern is repeated until the melting process finishes. We defined the Rayleigh number as $\mathbf{R a}=g \beta \Delta T L^{3} / \alpha v$ and average Nusselt number Nu as follows:

$$
\overline{N u}=-\int_{0}^{L} \frac{d T}{d x} d y
$$

where $\Delta T=T_{\mathbf{h}}-T_{m}$, and $T_{\mathbf{h}}$ is the hot wall temperature.

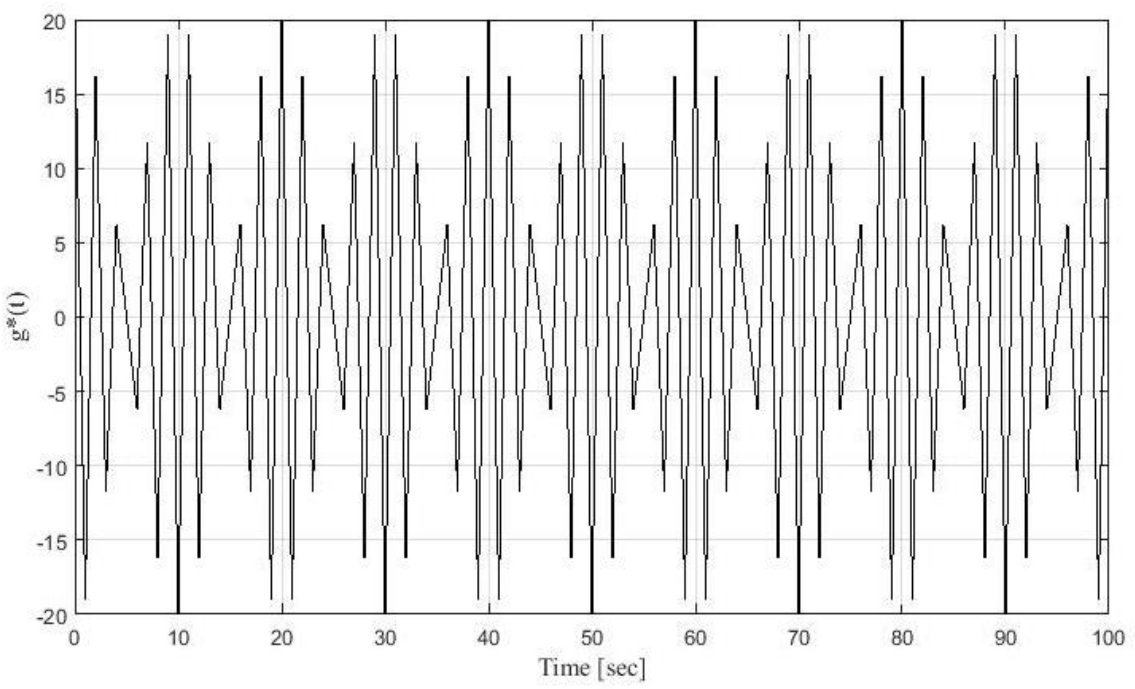

Fig. 2: Gravity oscillations versus time.

The boundary and initial conditions for the present problem are at the top and bottom:

$$
0 \leq x \leq R, y=0, L,=v=0, \frac{\partial T}{\partial y}=0
$$

at the cylinder wall: $\quad x=R, 0 \leq y \leq L, u=v=0, T=T_{h}$

at the symmetry line: $\quad x=0,0 \leq y \leq L, \frac{\partial u}{\partial x}=0, v=0, \frac{\partial T}{\partial x}=0$

The following section provides information on numerical scheme calculating the Nusselt number at the hot wall in this study.

\section{Numerical Procedure and Mesh Independency}

In this study, finite element method (FEM) was used to solve the governing equations. The direct solver method for two coupled modules (heat transfer in fluid and laminar flow) was utilized and the governing equations were solved numerically with the commercial COMSOL Multiphysics software (4.3b). The convergence for this solver was 
set to be $10^{-3}$ for termination criteria, and linear approximation was assumed within the elements. Non-uniform mesh distribution was considered with higher mesh along the hot surfaces for higher accuracy in results. In this simulation, we used different element sizes (different grid in $\mathrm{x}$ and $\mathrm{y}$ directions) and calculated the average Nusselt number at the hot surface. Fig. 3 shows the average Nusselt number calculated using Eq. (7). Based on Figure 3, the average Nusselt number did not change significantly beyond the total number of $25092(82 \times 306)$ elements. Thus, we used this number of elements for the rest of the calculation in this study.

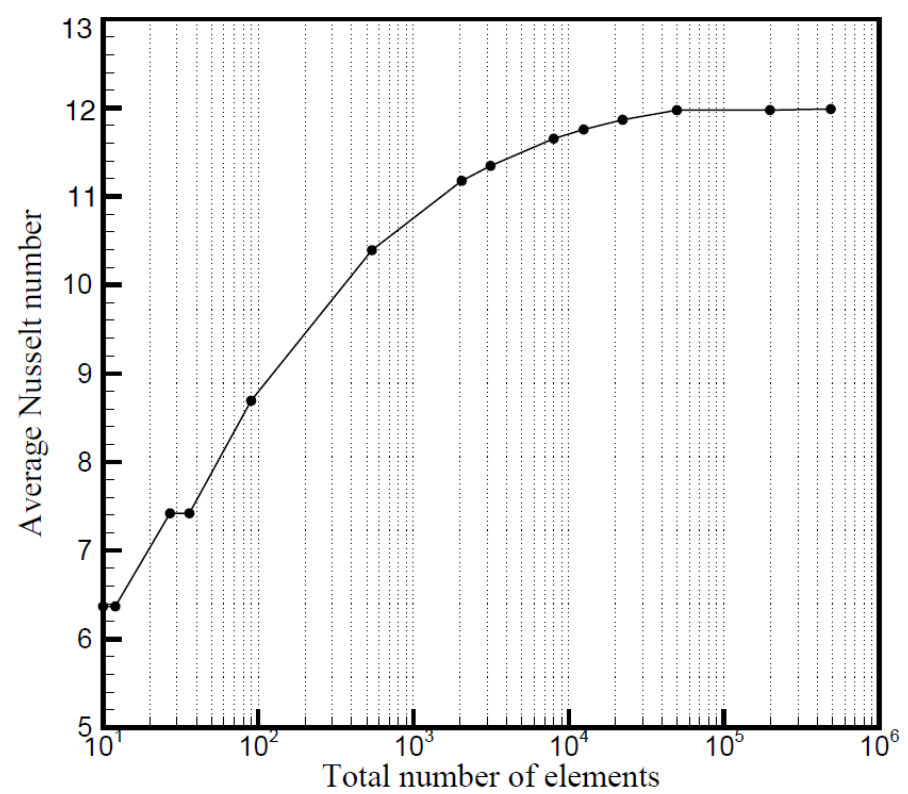

Fig. 3: Grid independency analysis on the enclosure.

\section{Results and Discussion}

Figure 3 shows the melting behaviour of PCM as a function of time when the vibration effect is absent. Initially the entire PCM is in the solid form at the initial temperature. Just after initiation of the higher wall temperature, the solid PCM absorbs the thermal energy, the temperature of the solid PCM near the outer surface of the cylinder starts to increase, reaches the melting point and starts to melt. Initially, at $100 \mathrm{~s}$, the melting happens equally along the outer perimeter and the solid-liquid interface approaches the centre of the enclosure. Conduction is the dominant mode of heat transfer in this stage. As melting progresses, at $200 \mathrm{~s}$, natural convection starts to dominate the heat transfer at the upper part of the enclosure due to stratification. Therefore, higher melting of PCM is observed at the top of the enclosure compared the bottom. At 300s, the PCM at the top of the enclosure is melted completely and the melting progresses in the downward direction until the entire PCM is melted. 


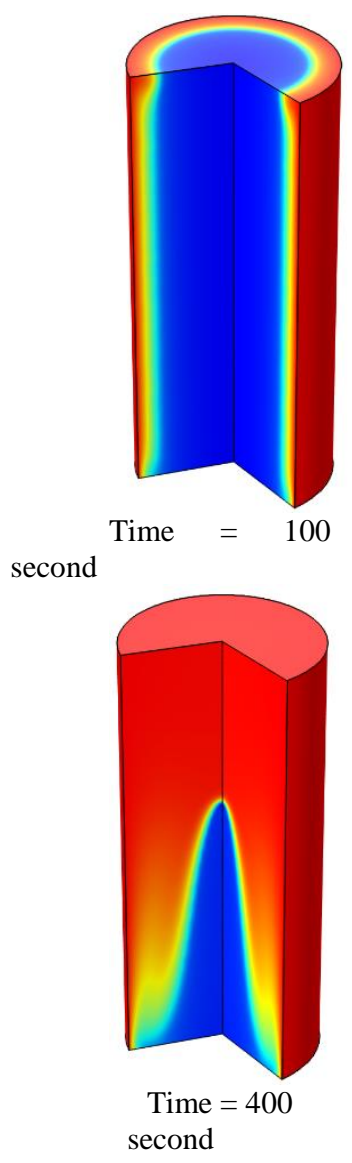

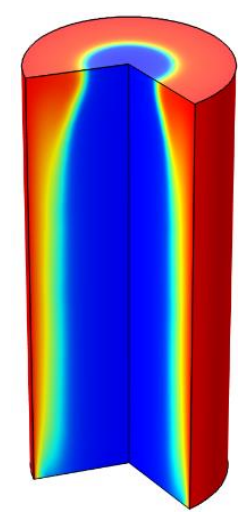

Time $=200$

second

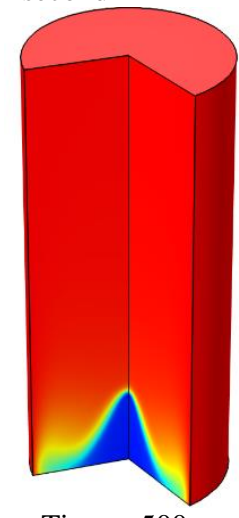

Time $=500$

second

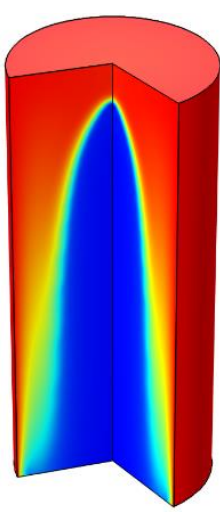

Time $=300$

second

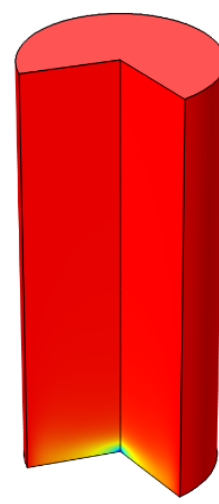

Time $=598$

second

Fig. 3: Temperature distribution of PCM inside the enclosure during melting process for different times having constant gravity.

Figure 4 shows the melting behaviour of PCM as a function of time when the vibration effect is present. The effect of vibration is not pronounced initially. At the beginning of melting, PCM melts equally along the outer surface and the melting progresses towards the centre. It has been found that as time progresses, the effect of vibration is becoming noticeable with melting both the top and bottom of the cylinder. The effect of stratification is not observed due to the presence of vibration. Therefore melting occurs almost at equal rate both at the top and bottom of the cylinder. 


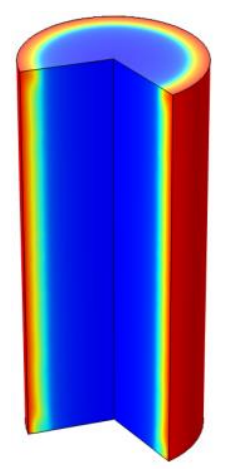

Time $=100$ second

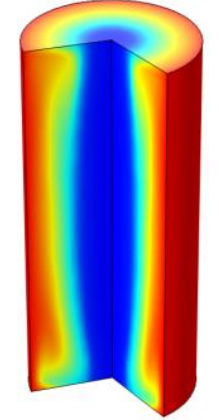

Time $=400$ second
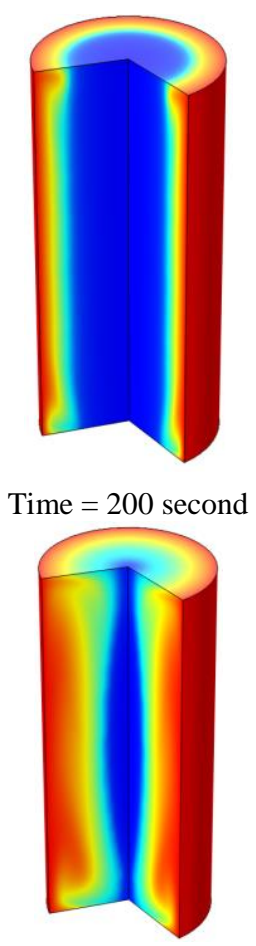

Time $=500$ second
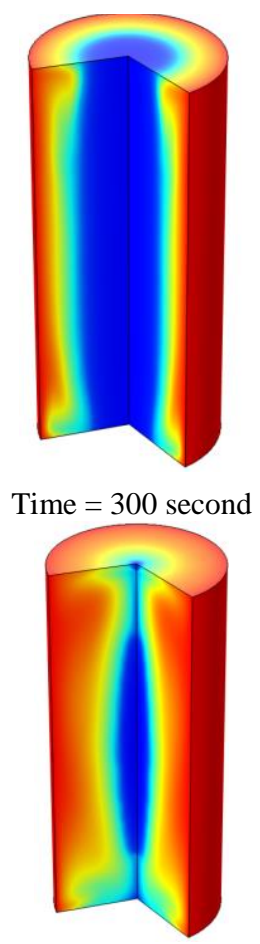

Time $=598$ second

Fig. 4: Temperature distribution of PCM inside the enclosure during melting process for different times having variable gravity function.

\section{Conclusion}

The melting behaviour of a PCM is numerically investigated due to the presence of vibration. This is a novel study. No study exists in the literature that considers the effect of vibration during melting of PCM. This is a very preliminary study. In future, the effect of amplitude and frequency of vibration on the melting will be considered numerically along with experimental measurements of melt fraction and energy transfer rates. This study is applicable to battery thermal management for the hybrid vehicles where PCM can be used for controlling the temperature of the battery.

\section{References}

[1] A. Sharma, et al, "Review on thermal energy storage with phase change materials and applications," Renewable and Sustainable energy reviews, vol. 13, no. 2 (2009): 318-345, 2009.

[2] S. Lorente, A. Bejan, and J. L. Niu, "Phase change heat storage in an enclosure with vertical pipe in the center," International Journal of Heat and Mass Transfer, vol. 72, pp. 329-335, 2014.

[3] M. Esen, A. Durmuş, and A. Durmuş, "Geometric design of solar-aided latent heat store depending on various parameters and phase change materials," Solar energy, vol. 62, no. 1, pp. 19-28, 1998.

[4] S. Kashani, et al, "Numerical analysis of melting of nano-enhanced phase change material in latent heat thermal energy storage system," Thermal Science, vol. 18, no. 2, pp. 335-345, 2014.

[5] A. Sciacovelli, F. Colella, and V. Verda, "Melting of PCM in a thermal energy storage unit: Numerical investigation and effect of nanoparticle enhancement," International Journal of Energy Research, vol. 37, no. 13, pp. 1610-1623, 2013.

[6] D. Singh, et al, "Analysis of a graphite foam- $\mathrm{NaCl}$ latent heat storage system for supercritical $\mathrm{CO} 2$ power cycles for concentrated solar power," Solar Energy, vol. 118, pp. 232-242, 2015.

[7] H. Liu, et al. "The melting of phase change material in a cylinder shell with hierarchical heat sink array," Applied Thermal Engineering, vol. 73, no. 1, pp. 975-983, 2014.

[8] J. N. W. Chiu, and V. Martin, "Multistage latent heat cold thermal energy storage design analysis," Applied energy, vol. 112, pp. 1438-1445, 2013.

[9] L. Bilir, and Z. İlken, "Total solidification time of a liquid phase change material enclosed in cylindrical/spherical 
containers," Applied thermal engineering, vol. 25, no. 10, pp. 1488-1502, 2005.

[10] S. F. Hosseinizadeh, A. A. Rabienataj Darzi, and F. L. Tan, "Numerical investigations of unconstrained melting of nano-enhanced phase change material (NEPCM) inside a spherical container," International Journal of Thermal Sciences, vol. 51, pp. 77-83, 2012.

[11] A. R. Archibold, et al, "The melting process of storage materials with relatively high phase change temperatures in partially filled spherical shells," Applied Energy, vol. 116, pp. 243-252, 2014.

[12] S. Karthikeyan, et al, "Parametric studies on packed bed storage unit filled with PCM encapsulated spherical containers for low temperature solar air heating applications," Energy conversion and management, vol. 78, pp. 74-80, 2014.

[13] H. Sattari, et al, "CFD simulation of melting process of phase change materials (PCMs) in a spherical capsule," International Journal of Refrigeration, vol. 73, pp. 209-218, 2017.

[14] S. Karthikeyan, and R. Velraj, "Numerical investigation of packed bed storage unit filled with PCM encapsulated spherical containers-a comparison between various mathematical models," International Journal of Thermal Sciences, vol. 60, pp. 153-160, 2012.

[15] A. R. Archibold, et al., "The effects of radiative heat transfer during the melting process of a high temperature phase change material confined in a spherical shell," Applied Energy, vol. 138, pp. 675-684, 2015.

[16] L. Yang, X. Zhang, and G. Xu, "Thermal performance of a solar storage packed bed using spherical capsules filled with PCM having different melting points," Energy and Buildings, vol. 68, pp. 639-646, 2014.

[17] K. A. R. Ismail, and J. R. Henriquez, "Solidification of PCM inside a spherical capsule," Energy conversion and management, vol. 41, no. 2, pp. 173-187, 2000. 